\title{
Single Impulsive Controller for Exponential Synchronization of Stochastic Lur'e Networks with Impulsive Disturbance
}

\author{
Yi Zhao, Ze Tang, and Jianwen Feng \\ College of Mathematics and Computational Sciences, Shenzhen University, Shenzhen 518060, China \\ Correspondence should be addressed to Jianwen Feng; fengjw@szu.edu.cn
}

Received 8 January 2013; Accepted 22 April 2013

Academic Editor: Jinde Cao

Copyright (C) 2013 Yi Zhao et al. This is an open access article distributed under the Creative Commons Attribution License, which permits unrestricted use, distribution, and reproduction in any medium, provided the original work is properly cited.

\begin{abstract}
The investigations are made on the exponential synchronization of the stochastic Lure system with nonlinear coupling and impulsive disturbance. The impulsive effects in complex networks could play a positive or a negative role for synchronization. For the sake of simplification and efficiency, a single impulsive controller is designed to realize the synchronization of the impulsive dynamical network with respect to stabilizing and destabilizing impulsive effects. Sufficient conditions are derived to guarantee the realization of the exponential synchronization for all initial values by means of the Lyapunov stability theorem, the comparison principle, and the linear matrix inequalities (LMIs). Numerical simulations are given to support the validity of the analytical results.
\end{abstract}

\section{Introduction}

For reasons that the synchronization of complex dynamical networks are ubiquity in both the natural and the artificial worlds and it can interpret the essence of the collective behavior in nature, it has attracted more and more attention of scientists and engineers from various fields such as sociology, biology, mathematics, and physics [1-9]. Synchronization can be understood as the adjustment of rhythms or coherence of states by interaction and realized via a sufficient information exchange among the nodes' interconnection in dynamical networks. It has many potential applications in such areas as biological systems [1], secure communications [3], information processing, mechanical engineering, identification and pattern recognition [4], and neuronal networks $[2,6,7,9]$.

Regarding the already known results on complex dynamical networks synchronization, there are three types of main communication methods between nodes normally considered: continuous coupling [7-9], intermittent communication [10], and impulsive exchange [11-14]. Impulsive exchange is a common phenomenon in many evolving networks. In the impulsive communication framework, the dynamics of each node is only affected by its neighbors at the impulsive instants and there exist impulsive effects in the dynamical behavior of nodes. For example, the states of electronic networks and biological networks are often subject to instantaneous disturbances and experience abrupt changes at certain instants, which may be caused by switching phenomenon, frequency change, or other sudden noise, which could be expressed as impulsive effects. Thus, impulsive dynamical networks, which involve sudden changes at certain discrete times, are receiving more and more attention of researchers for various fields, that is, [15]. The impulsive effects could play a positive or a negative role for synchronization of complex networks; see $[13,14]$ and the reference therein. Then, a natural question comes up when the researches about impulsive dynamical networks go deeper: How to design an efficient controller to make such networks achieve synchronization with respect to different types of impulsive effects? In particular, an impulsive controller, which means the information interchanging between nodes occurs impulsively only at some discrete time instants, proves that it can save the occupation of the communication channels. A rough idea is that when the impulsive effects are stabilizing, impulsive controllers could be used for synchronizing. Good prior works had been made on the synchronization of complex works with impulses controllers; see [11-13, 16] and the references therein. When the impulsive effects are destabilizing, feedback controllers should be involved to cancel some negative effects of impulsive disturbance. Mentions should be made that are in [14], the authors show that the complex networks with destabilizing impulses could reach a self-synchronized 
state if the impulses do not happen too frequently. However, the self-synchronized state depends on the network structure as well as the initial values of the nodes, which is difficult to predict. The previous research works motivate us to investigate the synchronization problem of complex networks via a single impulsive controller, in case the impulsive effects are stabilizing and destabilizing, respectively. With the help of a single controller, we are able to synchronize the complex networks to required states. Our work is complementary to the existing known results.

In this paper, the global and exponential synchronization problem of the Lur'e system will be discussed. The Lur'e systems, including the Goodwin model [17], repressilator [18], toggle switch [19], swarm model [20] and Chua's circuit [21], are a class of nonlinear systems which can be represented as a linear dynamical system with feedback interconnected to a nonlinearity satisfying a sector condition. Since the Lur'e system was first proposed by Lur'e and Postnikov, many researchers have attached much importance to the dynamics on such systems, and a large number of results have been obtained for the synchronization problem of the Lur'e system, such as [22-27]. Every node in the complex dynamical network studied in this paper is a Lur'e system; it has nonlinear local dynamical behaviors $A x_{i}(t)+B f\left(C x_{i}(t)\right)$ for the $i$ th node. Due to the instantaneous perturbations and abrupt change existing in many realistic networks, in order to fit with the real world, the stochastic phenomenon is going to be considered in the synchronization analysis; see [28]. The information interchange between each two nodes occurs impulsively at some certain impulsive instants but happens commonly at the nonimpulsive instants. Based on pinning impulsive control scheme, a single impulsive controller is imposed to the impulsive dynamical network with respect to different types of impulsive effects. Sufficient conditions are derived to guarantee the realization of the exponential synchronization pattern for all initial values by means of the Lyapunov stability theorem, the comparison principle, and linear matrix inequalities (LMIs). In addition, numerical simulations are given to support the validity of the main results.

The rest of the paper is organized as follows. In Section 2, we give some preparatory works, such as definitions and lemmas and next present the impulsive stochastic dynamical Lur'e network model. In Section 3, we analyze the exponential synchronization of the impulsive network by using a single impulsive controller when the impulsive effects are stabilizing and destabilizing, respectively. A numerical simulation is given to verify our theoretical results in Section 4 . We conclude the paper in Section 5.

Notations. The mark $A^{T}$ denotes the transport of the matrix A. $R^{n}$ denotes the $n$-dimensional Euclidean space. $R^{n \times n}$ are $n \times n$ real matrices. $\operatorname{diag}\{\cdots\}$ stands for a diagonal matrix. The sign $\|\cdot\|$ stands for the Euclid norm of the matrix or the vector. A symmetric real matrix $A$ is positive definite (semidefinite) if $x^{T} A x>0(\geq 0)$ for all nonzero $x$, we denote this as $A>0(A \geq 0)$. $I$ stands for the identity matrix with proper dimension. $\lambda_{\max }(\cdot)$ is used to denote the maximum eigenvalue of a real symmetric matrix. Let $E(\zeta)$ be the expectation value of $\zeta$. The dimension of these vectors and matrices will be cleared in the context.

\section{Preliminaries and Model Description}

In this section, some preliminaries such as definitions and lemmas will be firstly given, which are necessary throughout the paper. Then, we will present the impulsive dynamical network model with stochastic perturbations, which is composed of the Lur'e systems; it will be converted into the error impulsive dynamical network.

Definition 1. A dynamical network is said to realize global exponential synchronization, if there exist $\nu>0, T_{0}>0$ and $\theta>0$ such that for any initial values $\phi_{i}(\cdot)(i=1,2, \ldots, N)$,

$$
\left\|x_{i}(t)-x_{j}(t)\right\| \leq \theta e^{-v t}
$$

hold for all $t>T_{0}$, and for any $i, j=1,2, \ldots, N$.

Definition 2 (see [29]). The average impulsive interval of the impulsive sequence $\zeta=\left\{t_{1}, t_{2}, \ldots\right\}$ is less than $T_{a}$, if there exist a positive integer $N_{0}$ and a positive number $T_{a}$, such that

$$
N_{\zeta}(T, t) \geq \frac{T-t}{T_{a}}-N_{0}, \quad \forall T \geq t \geq 0,
$$

where $N_{\zeta}(T, t)$ denotes the number of impulsive times of the impulsive sequence $\zeta$ in the time interval $(t, T)$. As a consequence, the average impulsive interval of the impulsive sequence $\zeta=\left\{t_{1}, t_{2}, \ldots\right\}$ is not less than $T_{a}$, if there exist a positive integer $N_{0}$ and a positive number $T_{a}$, such that

$$
N_{\zeta}(T, t) \leq \frac{T-t}{T_{a}}+N_{0}, \quad \forall T \geq t \geq 0 .
$$

Lemma 3 (see [30]). Suppose $D=\left(d_{i j}\right) \in R^{n \times n}$, and it satisfies $d_{i j}=d_{j i}, d_{i i}=-\sum_{j=1, j \neq i}^{n} d_{i j}$; then, for any two vectors $x=$ $\left(x_{1}, x_{2}, \ldots, x_{n}\right)^{T} \in R^{n}, y=\left(y_{1}, y_{2}, \ldots, y_{n}\right)^{T} \in R^{n}$, one has

$$
x^{T} D y=-\sum_{j>i} d_{i j}\left(x_{j}-x_{i}\right)\left(y_{j}-y_{i}\right) .
$$

Lemma 4 ([31] comparison principle). Consider the following stochastic system with impulsive:

$$
\begin{aligned}
d x(t)= & \phi(t, x(t)) d t+\eta(t, x(t)) d w(t) \quad t \geq 0, t \neq t_{k}, \\
& x\left(t_{k}^{+}\right)-x\left(t_{k}^{-}\right)=U_{k}\left(x\left(t_{k}^{-}\right)\right) \quad k \in N .
\end{aligned}
$$

Assume that there exist a Lyapunov function $V(t, x(t))$ and functions $\varphi, \psi_{k}$ with $\varphi(t, 0)=\psi_{k}(0)=0$ for any $t \geq 0, k \in N$, such that:

(1) there exist positive constants $c_{1}, c_{2}$ such that for all $t \geq$ $t_{0}, c_{1}\|x(t)\| \leq V(t, x(t)) \leq c_{2}\|x(t)\| ;$

(2) there exists continuous function $\varphi: R^{+} \times R^{+} \rightarrow R$, and $\varphi(t, x(t))$ is concave on $s$ for each $t \in R^{+}$, such that $\mathscr{L} V(t, x)=V_{t}(t, x)+V_{x}(t, x) \varphi(t, x)+(1 / 2) \operatorname{trace}\left[\eta^{T}(t\right.$, $\left.x) V_{x x} \eta(t, x)\right]$ 
(3) there exist continuous and concave functions $\psi_{k}$ : $R^{+} \rightarrow R^{+}, k \in N$, such that $V\left(t_{k}^{+}, x\left(t_{k}^{+}\right)\right) \leq \psi_{k}\left(V\left(t_{k}^{-}\right.\right.$, $\left.\left.x\left(t_{k}^{-}\right)\right)\right)$;

then, the exponential stability of the trivial solution of the following comparison systems,

$$
\begin{gathered}
\dot{w}(t)=\phi(t, w(t)) \quad t \geq 0, t \neq t_{k}, \\
w\left(t_{k}^{+}\right)=\psi_{k}\left(w\left(t_{k}^{-}\right)\right) \quad k \in N, \\
w\left(t_{0}\right)=E\left(V\left(t_{0}, x_{0}\right)\right),
\end{gathered}
$$

implies the exponential stability of the trivial solution of the stochastic impulsive system (5).

Consider the following network model with nonlinear and asymmetrical coupling, and the nodes are composed of the Lur'e system:

$$
\dot{x}_{i}(t)=A x_{i}(t)+B \widetilde{f}\left(C x_{i}(t)\right)+c \sum_{j=1}^{N} l_{i j} \Gamma \widetilde{H}\left(x_{j}(t)\right)
$$

$$
i=1,2, \ldots, N \text {, }
$$

where $x_{i}(t)=\left[x_{i}^{1}(t), x_{i}^{2}(t), \ldots, x_{i}^{n}(t)\right]^{T} \in R^{n}$, for $i=1,2$, $\ldots, N . A \in R^{n \times n}, B \in R^{n \times m}, C \in R^{m \times n}$ are constant matrices. The constant $c>0$ denotes the coupling strength and $\Gamma=$ $\operatorname{diag}\left\{\gamma_{1}, \gamma_{2}, \ldots, \gamma_{n}\right\} \in R^{n \times n}$ is the inner-linking matrix, which is a diagonal matrix with $\gamma_{i} \geq 0$. Function $\widetilde{f}: R^{m} \rightarrow R^{m}$ is a memoryless nonlinear vector valued function which is continuously differentiable on $R, \widetilde{f}(0)=0$. Matrix $L=\left(l_{i j}\right) \in$ $R^{N \times N}$ is the coupling matrix, which indicates the connection topology, and it is decided by the network structure. $l_{i j}>0$ if there is a connection from node $i$ to node $j(i \neq j)$, otherwise $l_{i j}=0$ and it satisfies zero-sum-row condition, that is, $l_{i i}=$ $-\sum_{j \neq i j=1}^{N} l_{i j}$. We do not assume that $l_{i j}=l_{j i}$, which means that $L$ is an asymmetry matrix. The nonlinear coupling function $\widetilde{H}\left(x_{j}(t)\right)=\left(\tilde{h}\left(x_{j 1}(t)\right), \widetilde{h}\left(x_{j 2}(t)\right), \ldots, \tilde{h}\left(x_{j n}(t)\right)\right)^{T}$ satisfies the following conditions: $(\widetilde{h}(u)-\tilde{h}(v)) /(u-v) \geq \vartheta>0$ for any $u, v \in R$.

For many realistic complex networks, the state of node always suffers from instantaneous perturbations and is subject to abrupt change at certain instants. Taking those situations into consideration, we present the following stochastic impulsive dynamical Lur'e network with nonlinear coupling:

$$
\begin{aligned}
& d x_{i}(t)= {\left[A x_{i}(t)+B \tilde{f}\left(C x_{i}(t)\right)\right] d t+\tilde{g}\left(x_{i}(t), t\right) d w(t) } \\
&+c \sum_{j=1}^{N} l_{i j} \Gamma \widetilde{H}\left(x_{j}(t)\right) d t \quad t>0, t \neq t_{k}, \\
& x_{j}\left(t_{k}^{+}\right)-x_{i}\left(t_{k}^{+}\right)=\mu\left(x_{j}\left(t_{k}^{-}\right)-x_{i}\left(t_{k}^{-}\right)\right) \quad \text { for }(i, j): l_{i j}>0,
\end{aligned}
$$

where $w(t) \in R^{m}$ is an $m$-dimensional Brownian motion, $\tilde{g}: R^{n} \times R^{+} \rightarrow R^{n \times m}$ is the noise intensity function matrix satisfying $\widetilde{g}\left(t, 0^{n}\right)=0^{n \times m}$.
Remark 5. Throughout this paper, we always assume that $x_{i}(t)$ is left-hand continuous at $t=t_{k}$, that is, $x_{i}\left(t_{k}^{-}\right)=x_{i}\left(t_{k}\right)$. Therefore, according to the above assumption, we can get that the solutions of (8) are piecewise left-hand continuous functions with discontinuities at $t=t_{k}, k=1,2, \ldots$ On the other sides, from the second equation of (5), we can get that, in this paper, we focus on the impulsive effects (sudden changing) in the process of signal exchanging rather than the impulsive effects on the nodes' states.

In order to derive the main results of the stochastic impulsive dynamical Lur'e network, we make the following assumptions.

Assumption 6. The nonlinear function $\tilde{f}(\cdot)$ is assumed to satisfy a Lipschitz condition, that is, there exists a constant $\kappa>0$ such that $\|\tilde{f}(u)-\tilde{f}(v)\| \leq \kappa\|u-v\|$ holds for any $u, v \in R^{n}$.

Assumption 7. Assume that the noise intensity function matrix $\widetilde{p}: R^{n} \times R^{+} \rightarrow R^{n \times m}$ is uniformly Lipschitz continuous in terms of the norm induced by the trace inner product on the following matrices:

$$
\begin{aligned}
& \operatorname{trace} {\left[(\widetilde{g}(u(t), t)-\widetilde{g}(v(t), t))^{T} \cdot(\widetilde{g}(u(t), t)-\widetilde{g}(v(t), t))\right] } \\
& \leq\|M(u(t)-v(t))\|^{2},
\end{aligned}
$$

for any $u, v \in R^{n}$, where $M$ is a known constant matrix with compatible dimensions.

\section{Synchronization Analysis for the Stochastic Lur'e System under a Single Controller}

In this section, we will investigate the global and exponential synchronization of the Lur'e system with stochastic perturbations. Define the error vectors as $e_{i}(t)=x_{i}(t)-s(t), i=$ $1,2, \ldots, N$, where $s(t)=\left[s^{1}(t), s^{2}(t), \ldots, s^{n}(t)\right] \in R^{n}$ is the solution of an isolated node in the Lur'e network satisfying

$$
d s(t)=[A s(t)+B \tilde{f}(C s(t))] d t+\widetilde{g}(s(t), t) d w(t),
$$

with initial condition $s_{0} \in R^{n}$. In this paper, $s(t)$ is the objective trajectory that the nonlinear stochastic impulsive dynamical network (8) will be forced to.

For $|\mu|<1$ in (8), which means that the corresponding impulsive effects are synchronizing, we propose a single impulsive controller to the network. Without loss of generality, the first node is selected to be controlled. The controlled impulsive dynamical Lur'e network is obtained as follows:

$$
\begin{gathered}
d e_{i}(t)=\left[A e_{i}(t)+B f\left(C e_{i}(t)\right)\right] d t+g\left(x_{i}(t), t\right) d w(t) \\
+c \sum_{j=1}^{N} l_{i j} \Gamma H\left(e_{j}(t)\right) d t \quad t>0, t \neq t_{k}, \\
e_{j}\left(t_{k}^{+}\right)-e_{i}\left(t_{k}^{+}\right)=\mu\left(e_{j}\left(t_{k}^{-}\right)-e_{i}\left(t_{k}^{-}\right)\right) \text {for }(i, j): l_{i j}>0, \\
e_{1}\left(t_{k}^{+}\right)=\mu e_{1}\left(t_{k}^{-}\right),
\end{gathered}
$$


where

$$
\begin{gathered}
f\left(C e_{i}(t)\right)=\tilde{f}\left(C x_{i}(t)\right)-\tilde{f}(C s(t)), \\
g\left(e_{i}(t), t\right)=\widetilde{g}\left(x_{i}(t), t\right)-\widetilde{g}(s(t), t), \\
H\left(e_{i}(t)\right)=\left[h\left(e_{i 1}(t)\right), h\left(e_{i 2}(t)\right), \ldots, h\left(e_{i n}(t)\right)\right]^{T}, \\
h\left(e_{i j}(t)\right)=\widetilde{h}\left(x_{i j}(t)\right)-\widetilde{h}(s(t)),
\end{gathered}
$$

for $i=1,2, \ldots, N ; j=1,2, \ldots, n$. Since $(\widetilde{h}(u)-\widetilde{h}(v)) /(u-v) \geq$ $\vartheta>0$, we have $(h(u)-h(v)) /(u-v) \geq \vartheta>0$.

In the following part, we are devoted to studying the global exponential stability for the controlled impulsive stochastic dynamical Lur'e network (3). Suppose that $\xi=$ $\left(\xi_{1}, \xi_{2}, \ldots, \xi_{N}\right)^{T}$ is the normalized left eigenvector of the configuration matrix $L$ with respect to the eigenvalue 0 satisfying $\|\xi\|=1$. Since the coupling matrix $L$ is irreducible, $\xi_{i}>0$ for $i=1,2, \ldots, N$. Let $\Xi=\operatorname{diag}\left\{\xi_{1}, \xi_{2}, \ldots, \xi_{N}\right\}>0$.

Theorem 8. Consider the controlled impulsive stochastic dynamical Lur'e network (3) when $|\mu|<1$. Suppose that Assumptions 6 and 7 hold, and the average impulsive interval of the impulsive sequence $\zeta=\left\{t_{1}, t_{2}, \ldots\right\}$ is less than $T_{a}$. Then, the controlled impulsive stochastic dynamical network (3) is globally exponentially stable if

$$
\frac{2 \ln (|\mu|)}{T_{a}}+\delta<0,
$$

where $\delta=\lambda_{\max }\left(A+A^{T}+M^{T} M\right)+2 \kappa \sqrt{\lambda_{\max }\left(B^{T} B\right) \lambda_{\max }\left(C^{T} C\right)}$.

Proof. Construct a Lyapunov function candidate in the form of

$$
V(t)=\sum_{i=1}^{N} \xi_{i} e_{i}^{T}(t) e_{i}(t) .
$$

For $t \in\left(t_{k-1}, t_{k}\right], k=1,2, \ldots$, we have

$$
\begin{aligned}
\mathscr{L} V(t)= & 2 \sum_{i=1}^{N} \xi_{i} e_{i}^{T}(t)\left\{A e_{i}(t)+B f\left(C e_{i}(t)\right)\right. \\
& \left.+c \sum_{j=1}^{N} l_{i j} \Gamma H\left(e_{j}(t)\right)\right\} \\
& +\sum_{i=1}^{N} \operatorname{trace}\left[g^{T}\left(e_{i}(t), t\right) \Xi g\left(e_{i}(t), t\right)\right] \\
\leq & 2 \sum_{i=1}^{N} \xi_{i} e_{i}^{T}(t)\left[A e_{i}(t)+B f\left(C e_{i}(t)\right)\right] \\
& +2 c \sum_{i=1}^{N} \xi_{i} \sum_{j=1}^{N} l_{i j} e_{i}^{T}(t) \Gamma H\left(e_{j}(t)\right) \\
& +\sum_{i=1}^{N} \operatorname{trace}\left[g^{T}\left(e_{i}(t), t\right) g\left(e_{i}(t), t\right)\right] .
\end{aligned}
$$

By Assumptions 6 and 7, the following inequalities can be obtained:

$$
\begin{aligned}
2 e_{i}^{T}(t) & B f\left(C e_{i}(t)\right) \\
\leq & 2\left\|e_{i}(t)\right\| \cdot\left\|B f\left(C e_{i}(t)\right)\right\| \\
& 2\left\|e_{i}(t)\right\| \cdot \sqrt{f^{T}\left(C\left(e_{i}(t)\right)\right) B^{T} B f\left(C e_{i}(t)\right)} \\
& \leq 2\left\|e_{i}(t)\right\| \cdot \sqrt{\lambda_{\max }\left(B^{T} B\right)} \cdot\left\|f\left(C e_{i}(t)\right)\right\| \\
& \leq 2 \sqrt{\lambda_{\max }\left(B^{T} B\right)}\left\|e_{i}(t)\right\| \cdot \kappa \sqrt{\left\|C e_{i}(t)\right\|^{2}} \\
& =2 \sqrt{\lambda_{\max }\left(B^{T} B\right)} \kappa\left\|e_{i}(t)\right\| \cdot \sqrt{e_{i}^{T}(t) C^{T} C e_{i}(i)} \\
& \leq 2 \kappa \sqrt{\lambda_{\max }\left(B^{T} B\right)} \sqrt{\lambda_{\max }\left(C^{T} C\right) e_{i}^{T}(t) e_{i}(t),} \\
\operatorname{trace}[ & \left.p^{T}\left(e_{i}(t), t\right) p\left(e_{i}(t), t\right)\right] \leq\left\|M e_{i}(t)\right\|^{2} \\
= & =e_{i}^{T}(t) M^{T} M e_{i}(t) .
\end{aligned}
$$

Let $e^{k}(t)=\left[e_{1 k}(t), e_{2 k}, \ldots, e_{N k}(t)\right]^{T}$ and $Q=\Xi L+L^{T} \Xi=$ $\left(q_{i j}\right)_{N \times N}$. Note that $Q$ is a symmetric irreducible matrix with zero row sum. We have

$$
\begin{aligned}
2 c \sum_{i=1}^{N} \xi_{i} \sum_{j=1}^{N} l_{i j} e_{i}^{T}(t) \Gamma H\left(e_{j}(t)\right) \\
=2 c \sum_{i=1}^{N} \xi_{i} \sum_{j=1}^{N} l_{i j} \sum_{k=1}^{n} e_{i k}(t) \gamma_{k} h\left(e_{j k}(t)\right) \\
=2 c \sum_{k=1}^{n} \gamma_{k} \sum_{i=1}^{N} \sum_{j=1}^{N} e_{i k}(t) l_{i j} h\left(e_{j k}(t)\right) \\
=2 c \sum_{k=1}^{n} \gamma_{k} e^{k}(t)^{T} Q h\left(e^{k}(t)\right) \\
=-c \sum_{k=1}^{n} \gamma_{k} \sum_{i=1}^{N} \sum_{j=1}^{N} q_{i j}\left(e_{i k}(t)-e_{j k}(t)\right) \\
\quad \times h\left(e_{i k}(t)-h\left(e_{j k}(t)\right)\right) \\
\leq-c \vartheta \sum_{k=1}^{n} \gamma_{k} \sum_{i=1}^{N} \sum_{j=1}^{N} q_{i j}\left(e_{i k}(t)-e_{j k}(t)\right)^{2} \leq 0 .
\end{aligned}
$$

Combining (16) and (17) gives

$$
\mathscr{L} V(t) \leq \delta V(t)
$$

for $t \in\left(t_{k-1}, t_{k}\right)$.

In the second equation in the system (8), $x_{j}\left(t_{k}^{+}\right)-x_{i}\left(t_{k}^{+}\right)=$ $\mu\left(x_{j}\left(t_{k}^{-}\right)-x_{i}\left(t_{k}^{-}\right)\right)$for any pair of $(i, j)$ which satisfies $l_{i j}>$ 0 . Based on the coupling matrix $L$, is irreducible; we have assumed yet, for any suffix $i(i \neq 1)$, there exist a reachable 
route between the 1-node and the $i$-node even though the route is not limited in one and immediate. That is, there exist suffixes $\tau_{1}, \tau_{1}, \ldots, \tau_{m}$ such that $l_{1, \tau_{1}}>0, l_{\tau_{1}, \tau_{2}}>0, \ldots, l_{\tau_{m}, i}>0$. Hence, from the analysis, for the pair of suffixes 1 and $i$ ( $i=$ $2,3, \ldots, N)$, we obtain

$$
\begin{aligned}
x_{i}\left(t_{k}^{+}\right)- & x_{1}\left(t_{k}^{+}\right) \\
= & {\left[x_{i}\left(t_{k}^{+}\right)-x_{\tau_{m}}\left(t_{k}^{+}\right)\right]+\left[x_{\tau_{m}}\left(t_{k}^{+}\right)-x_{\tau_{m-1}}\left(t_{k}^{+}\right)\right] } \\
& +\cdots+\left[x_{\tau_{1}}\left(t_{k}^{+}\right)-x_{1}\left(t_{k}^{+}\right)\right] \\
= & \mu\left[x_{i}\left(t_{k}^{-}\right)-x_{\tau_{m}}\left(t_{k}^{-}\right)\right]+\mu\left[x_{\tau_{m}}\left(t_{k}^{-}\right)-x_{\tau_{m-1}}\left(t_{k}^{-}\right)\right] \\
& +\cdots+\mu\left[x_{\tau_{1}}\left(t_{k}^{-}\right)-x_{1}\left(t_{k}^{-}\right)\right] \\
= & \mu\left[x_{i}\left(t_{k}^{-}\right)-x_{\tau_{m}}\left(t_{k}^{-}\right)+x_{\tau_{m}}\left(t_{k}^{-}\right)-x_{\tau_{m-1}}\left(t_{k}^{-}\right)\right. \\
& \left.+\cdots+x_{\tau_{1}}\left(t_{k}^{-}\right)-x_{1}\left(t_{k}^{-}\right)\right] \\
= & \mu\left[x_{i}\left(t_{k}^{-}\right)-x_{1}\left(t_{k}^{-}\right)\right] .
\end{aligned}
$$

Recall the definition of the error vectors, we get $x_{i}\left(t_{k}^{+}\right)-s(t)=$ $\mu\left(x_{i}\left(t_{k}^{-}\right)-s(t)\right)$, that is

$$
e_{i}\left(t_{k}^{+}\right)=\mu e_{i}\left(t_{k}^{-}\right) .
$$

Therefore, when $t=t_{k}, k \in N$, one gets that

$$
\begin{aligned}
V\left(t_{k}^{+}\right) & =\sum_{i=1}^{N} e_{i}^{T}\left(t_{k}^{+}\right) e_{i}\left(t_{k}^{+}\right) \\
& =\mu^{2} \sum_{i=1}^{N} e_{i}^{T}\left(t_{k}^{-}\right) e_{i}\left(t_{k}^{-}\right)=\mu^{2} V\left(t_{k}^{-}\right) .
\end{aligned}
$$

By (18) and (21), we can obtain the following comparison system for the controlled impulsive stochastic dynamical Lur'e network (3):

$$
\begin{gathered}
\dot{w}(t)=\delta w(t) \quad t \geq 0, t \neq t_{k}, \\
w\left(t_{k}^{+}\right)=\mu^{2} w\left(t_{k}^{-}\right) \quad \mu^{2} \in(0,1), \quad k \in N, \\
w\left(t_{0}\right)=E\left(V\left(t_{0}\right)\right) .
\end{gathered}
$$

For $t \in\left(t_{0}, t_{1}\right]$,

$$
w(t)=e^{\delta\left(t-t_{0}\right)} w\left(t_{0}^{+}\right), \quad w\left(t_{1}^{-}\right)=e^{\delta\left(t_{1}-t_{0}\right)} w\left(t_{0}^{+}\right) .
$$

For $t \in\left(t_{1}, t_{2}\right]$,

$$
\begin{gathered}
w\left(t_{1}^{+}\right)=\mu^{2} w\left(t_{1}^{-}\right)=\mu^{2} e^{\delta\left(t_{1}-t_{0}\right)} E\left(V\left(t_{0}\right)\right), \\
w(t)=e^{\delta\left(t-t_{1}\right)} w\left(t_{1}^{+}\right)=e^{\delta\left(t-t_{1}\right)} \mu^{2} w\left(t_{1}^{-}\right) \\
=e^{\delta\left(t-t_{1}\right)} e^{\delta\left(t-t_{0}\right)} \mu^{2} w\left(t_{0}^{+}\right), \\
w\left(t_{2}^{-}\right)=\mu^{2} e^{\delta\left(t_{2}-t_{0}\right)} E\left(V\left(t_{0}\right)\right) .
\end{gathered}
$$

Similarly, for $t \in\left(t_{k-1}, t_{k}\right]$,

$$
\begin{aligned}
w(t) & =e^{\delta\left(t-t_{k-1}\right)} w\left(t_{k-1}^{+}\right)=e^{\delta\left(t-t_{k-1}\right)} \mu^{2} w\left(t_{k-1}\right) \\
& =e^{\delta\left(t-t_{k-1}\right)} \mu^{2} \mu^{2(k-1)} e^{\delta\left(t_{k-1}-t_{0}\right)} w\left(t_{0}^{+}\right) \\
& =e^{\delta\left(t-t_{0}\right)} \mu^{2 k} E\left(V\left(t_{0}\right)\right) .
\end{aligned}
$$

Based on the above analysis, for any $t>0$, one has

$$
\begin{aligned}
w(t) & =e^{\delta\left(t-t_{0}\right)} \mu^{2 k} E\left(V\left(t_{0}\right)\right) \\
& =e^{\delta\left(t-t_{0}\right)} \mu^{2 N_{\zeta}\left(t, t_{0}\right)} E\left(V\left(t_{0}\right)\right) \\
& \leq e^{\delta\left(t-t_{0}\right)} \mu^{2\left(\left(t-t_{0}\right) / T_{a}\right)-2 N_{0}} E\left(V\left(t_{0}\right)\right) \\
& =\mu^{-2 N_{0}} e^{\left(\left(2 \ln |\mu| / T_{a}\right)+\delta\right)\left(t-t_{0}\right)} E\left(V\left(t_{0}\right)\right) .
\end{aligned}
$$

By Lemma 4, it can be concluded that the controlled impulsive stochastic dynamical Lure network (3) is exponentially stable, which can further imply that the dynamical network (7) can be exponentially stabilized to the objective trajectory $s(t)$ by only imposing a single impulsive controller on the first node. Theorem 8 is proved completely.

Remark 9. It means that the nonlinear and asymmetric coupled impulsive stochastic dynamical Lur'e network (8) can be globally and exponentially controlled to the objective trajectory $s(t)$ by using a single impulsive controller. In the proving process, the Lyapunov stability theorem and the comparison principle are combined to derive the synchronization criteria for the impulsive stochastic dynamical Lur'e network by using a single impulsive controller. In fact, the node to be controlled can be selected randomly. In this paper, without loss of generality, we chose the first node as the impulsive controller was imposed to. It has been proved that the impulsive stochastic dynamical Lur'e network can achieve synchronization by only applying a single controller, not to speak of more controllers.

As a special case of the main result, we give the following stochastic impulsive dynamical Lure network with linear coupling:

$$
\begin{gathered}
d x_{i}(t)=\left[A x_{i}(t)+B \tilde{f}\left(C x_{i}(t)\right)\right] d t+\widetilde{g}\left(x_{i}(t), t\right) d w(t) \\
+c \sum_{j=1}^{N} l_{i j} \Gamma x_{j}(t) d t \quad t>0, t \neq t_{k}, \\
x_{j}\left(t_{k}^{+}\right)-x_{i}\left(t_{k}^{+}\right)=\mu\left(x_{j}\left(t_{k}^{-}\right)-x_{i}\left(t_{k}^{-}\right)\right) \text {for }(i, j): l_{i j}>0 .
\end{gathered}
$$

Correspondingly, we have the following conclusion for the stochastic impulsive dynamical Lur'e network with linear coupling.

Corollary 10. Consider the impulsive stochastic dynamical Lur'e network (27) under the same impulsive controller imposed on the first node. Suppose that Assumptions 6 and 7 hold, and the average impulsive interval of the impulsive sequence $\zeta=\left\{t_{1}\right.$, $\left.t_{2}, \ldots\right\}$ is less than $T_{a}$. Then, the impulsive stochastic dynamical 
network (27) with a single impulsive controller is globally exponentially stable, if the following condition holds:

$$
\begin{aligned}
& \frac{2 \ln |\mu|}{T_{a}}+\lambda_{\max }\left(A+A^{T}+M^{T} M\right) \\
& +2 \kappa \sqrt{\lambda_{\max }\left(B^{T} B\right) \lambda_{\max }\left(C^{T} C\right)}<0 .
\end{aligned}
$$

This means that the impulsive stochastic dynamical Lur'e network (27) can be globally and exponentially controlled to the objective trajectory $s(t)$ by using a single impulsive controller.

If we consider the following impulsive dynamical Lure network with linear coupling without stochastic distortions

$$
\begin{aligned}
d x_{i}(t)= & {\left[A x_{i}(t)+B \tilde{f}\left(C x_{i}(t)\right)\right] d t } \\
& +c \sum_{j=1}^{N} l_{i j} \Gamma x_{j}(t) d t \quad t>0, t \neq t_{k}, \\
x_{j}\left(t_{k}^{+}\right)-x_{i}\left(t_{k}^{+}\right)= & \mu\left(x_{j}\left(t_{k}^{-}\right)-x_{i}\left(t_{k}^{-}\right)\right) \text {for }(i, j): l_{i j}>0,
\end{aligned}
$$

we have another corollary, we give it in the form of the following.

Corollary 11. Consider the impulsive dynamical Lur'e network (29). Suppose that Assumptions 6 and 7 hold, and the average impulsive interval of the impulsive sequence $\zeta=\left\{t_{1}, t_{2}, \ldots\right\}$ is less than $T_{a}$. Then, the impulsive dynamical network (32) with a single impulsive controller is globally exponentially stable, if the following condition holds:

$$
\frac{2 \ln |\mu|}{T_{a}}+\lambda_{\max }\left(A+A^{T}\right)+2 \kappa \sqrt{\lambda_{\max }\left(B^{T} B\right) \lambda_{\max }\left(C^{T} C\right)}<0 .
$$

This means that the impulsive dynamical Lure network (29) can be globally and exponentially controlled to the objective trajectory $s(t)$ by using a single impulsive controller.

Remark 12. From Theorem 8 and its corollaries, it can be seen that condition (13), namely,

$$
\begin{aligned}
& \frac{2 \ln (|\mu|)}{T_{a}}+\lambda_{\max }\left(A+A^{T}+M^{T} M\right) \\
& +2 \kappa \sqrt{\lambda_{\max }\left(B^{T} B\right) \lambda_{\max }\left(C^{T} C\right)}<0,
\end{aligned}
$$

can be realized with the help of the term $2 \ln (|\mu|) / T_{a}$, which is negative once $|\mu|<1$. It corresponds to the observation that the impulsive effects are synchronizing. However, sometimes impulses can play a negative role for the synchronization of dynamical networks. We label this case as $|\mu|>1$ in (8). Then, with a rough idea, the term $2 \ln (|\mu|) / T_{a}>0$ can play a negative effect to realize condition (13), though a few of the systems (8) can still achieve synchronization under (13). We need to add a single feedback controller to cancel the desynchronization effects caused by impulses if $|\mu|>1$, which is an effective way to drive the system (8) into desirable synchronization states.
When $|\mu|>1$, after adding the feedback controller $u(t)=$ $-c l \Gamma e_{1}(t)$ to the first node of network (3), we have

$$
\begin{gathered}
d e_{1}(t)=\left[A e_{1}(t)+B f\left(C e_{1}(t)\right)\right] d t+g\left(x_{i}(t), t\right) d w(t) \\
+c \sum_{j=1}^{N} l_{1 j} \Gamma H\left(e_{j}(t)\right) d t-c l \Gamma e_{1}(t) d t, \\
t>0, t \neq t_{k}, \\
d e_{i}(t)=\left[A e_{i}(t)+B f\left(C e_{i}(t)\right)\right] d t+g\left(x_{i}(t), t\right) d w(t) \\
+c \sum_{j=1}^{N} l_{i j} \Gamma H\left(e_{j}(t)\right) d t, \quad t>0, t \neq t_{k}, i \geq 2, \\
e_{j}\left(t_{k}^{+}\right)-e_{i}\left(t_{k}^{+}\right)=\mu\left(e_{j}\left(t_{k}^{-}\right)-e_{i}\left(t_{k}^{-}\right)\right), \quad \text { for }(i, j): l_{i j}>0, \\
e_{1}\left(t_{k}^{+}\right)=\mu e_{1}\left(t_{k}^{-}\right) .
\end{gathered}
$$

Define the Laplacian matrix $\widetilde{L}=\left(\widetilde{l}_{i j}\right)_{N \times N}$ as follows:

$$
\tilde{l}_{i j}= \begin{cases}l_{i j}-\frac{l}{\vartheta}, & i=j=1, \\ l_{i j}, & \text { otherwise. }\end{cases}
$$

Let $\gamma_{\min }=\min \left\{\gamma_{1}, \gamma_{2}, \ldots, \gamma_{n}\right\}$, where $\gamma_{i}$ is the diagonal elements of $\Gamma$, and $\widetilde{Q}=\left(\widetilde{q}_{i j}\right)_{N \times N}=\Xi \widetilde{Q}+\widetilde{Q}^{T} \Xi$.

Theorem 13. Consider the controlled impulsive stochastic dynamical Lur'e network (3) when $|\mu|>1$. Suppose that Assumptions 6 and 7 hold, and the average impulsive interval of the impulsive sequence $\zeta=\left\{t_{1}, t_{2}, \ldots\right\}$ is not less than $T_{a}$. Then, the controlled impulsive stochastic dynamical network (3) is globally exponentially stable if

$$
\frac{2 \ln (|\mu|)}{T_{a}}+\widetilde{\delta}<0,
$$

where $\widetilde{\delta}=\lambda_{\max }\left(A+A^{T}+M^{T} M\right)+2 \kappa \sqrt{\lambda_{\max }\left(B^{T} B\right) \lambda_{\max }\left(C^{T} C\right)}+$ $c \vartheta \gamma_{\min } \lambda_{\max }(\widetilde{Q})$.

Proof. Consider a Lyapunov function as follows:

$$
V(t)=\sum_{i=1}^{N} \xi_{i} e_{i}^{T}(t) e_{i}(t) .
$$

For $t \in\left(t_{k-1}, t_{k}\right], k=1,2, \ldots$, we have

$$
\begin{aligned}
\mathscr{L} V(t)= & 2 \sum_{i=1}^{N} \xi_{i} e_{i}^{T}(t) \\
& \times\left\{A e_{i}(t)+B f\left(C e_{i}(t)\right)\right. \\
& \left.+c \sum_{j=1}^{N} l_{i j} \Gamma H\left(e_{j}(t)\right)-c l \Gamma e_{1}(t)\right\} \\
& +\sum_{i=1}^{N} \operatorname{trace}\left[g^{T}\left(e_{i}(t), t\right) \Xi g\left(e_{i}(t), t\right)\right]
\end{aligned}
$$




$$
\begin{aligned}
\leq & 2 \sum_{\mathrm{i}=1}^{N} \xi_{i} e_{i}^{T}(t)\left[A e_{i}(t)+B f\left(C e_{i}(t)\right)\right] \\
& +2 c \sum_{i=1}^{N} \xi_{i} \sum_{j=1}^{N} l_{i j} e_{i}^{T}(t) \Gamma H\left(e_{j}(t)\right) \\
& -2 c \sum_{i=1}^{N} \xi_{i} l e_{i}^{T}(t) \Gamma e_{1}(t) \\
& +\sum_{i=1}^{N} \operatorname{trace}\left[g^{T}\left(e_{i}(t), t\right) g\left(e_{i}(t), t\right)\right] .
\end{aligned}
$$

Note that all the eigenvalues of $\widetilde{Q}$ are negative; we have

$$
\begin{aligned}
& 2 c \sum_{i=1}^{N} \xi_{i} \sum_{j=1}^{N} l_{i j} e_{i}^{T}(t) \Gamma H\left(e_{j}(t)\right) \\
& =2 c \sum_{i=1}^{N} \xi_{i} \sum_{j=1}^{N} l_{i j} \sum_{k=1}^{n} e_{i k}(t) \gamma_{k} h\left(e_{j k}(t)\right) \\
& -2 c \sum_{i=1}^{N} \xi_{i} l e_{i}^{T}(t) \Gamma e_{1}(t) \\
& =2 c \sum_{k=1}^{n} \gamma_{k} \sum_{i=1}^{N} \sum_{j=1}^{N} e_{i k}(t) l_{i j} h\left(e_{j k}(t)\right) \\
& -2 c \sum_{i=1}^{N} \xi_{i} l_{i}^{T}(t) \Gamma e_{1}(t) \\
& =c \sum_{k=1}^{n} \gamma_{k} e^{k}(t)^{T} \mathrm{Qh}\left(e^{k}(t)\right) \\
& -2 c \sum_{i=1}^{N} \xi_{i} l e_{i}^{T}(t) \Gamma e_{1}(t) \\
& =-c \sum_{k=1}^{n} \gamma_{k} \sum_{i=1}^{N} \sum_{j=1}^{N} q_{i j}\left(e_{i k}(t)-e_{j k}(t)\right) \\
& \times h\left(e_{i k}(t)-h\left(e_{j k}(t)\right)\right) \\
& -2 c \sum_{i=1}^{N} \xi_{i} l e_{i}^{T}(t) \Gamma e_{1}(t) \\
& \leq-c \vartheta \sum_{k=1}^{n} \gamma_{k} \sum_{i=1}^{N} \sum_{j=1}^{N} q_{i j}\left(e_{i k}(t)-e_{j k}(t)\right)^{2} \\
& -2 c \sum_{i=1}^{N} \xi_{i} l e_{i}^{T}(t) \Gamma e_{1}(t) \\
& =c \vartheta \sum_{k=1}^{n} \gamma_{k}\left[e^{k}(t)^{T} \widetilde{Q} e^{k}(t)\right]
\end{aligned}
$$

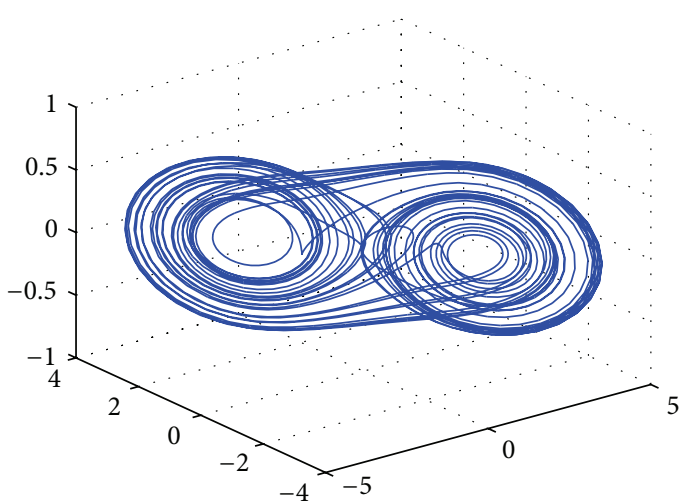

(a)

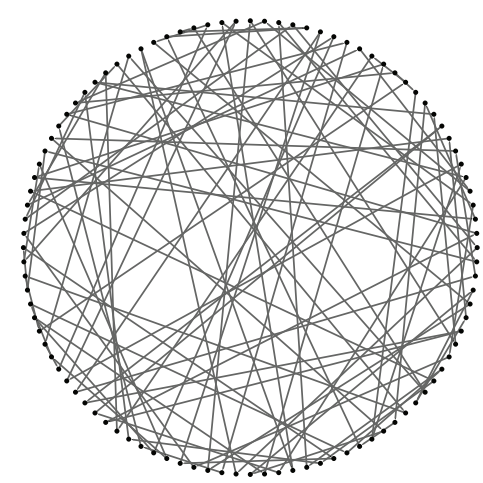

(b)

FIgURE 1: Phase trajectories of Chua's circuit without Brownian noise (a) and the network structure (b).

$$
\begin{aligned}
& \leq c \vartheta \lambda_{\max }(\widetilde{Q}) \sum_{k=1}^{n} \gamma_{k}\left(e^{k}(t)\right)^{T} e^{k}(t) \\
& \leq c \vartheta \gamma_{\min } \lambda_{\max }(\widetilde{Q}) \sum_{k=1}^{n}\left(e^{k}(t)\right)^{T} e^{k}(t) \\
& =c \vartheta \gamma_{\min } \lambda_{\max }(\widetilde{Q}) \sum_{i=1}^{N} e_{i}(t)^{T} e_{i}(t) \\
& \leq c \vartheta \gamma_{\min } \lambda_{\max }(\widetilde{Q}) \sum_{i=1}^{N} \xi_{i} e_{i}(t)^{T} e_{i}(t) \\
& =c \vartheta \gamma_{\min } \lambda_{\max }(\widetilde{Q}) V(t) .
\end{aligned}
$$

For the remaining parts in (36), we can use similar analysis in (16). Then, it could be obtained that

$$
\mathscr{L} V(t) \leq \tilde{\delta} V(t)
$$

for $t \in\left(t_{k-1}, t_{k}\right)$. 


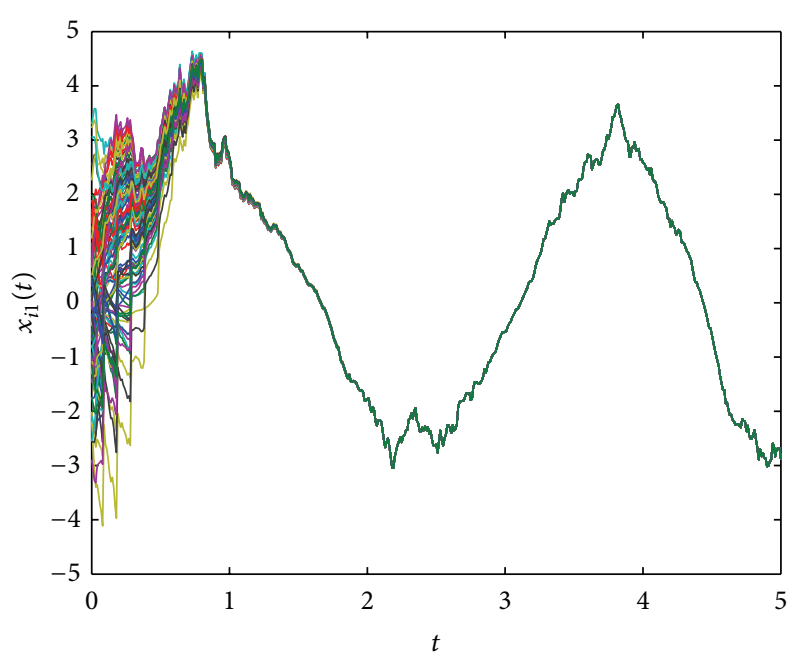

(a)

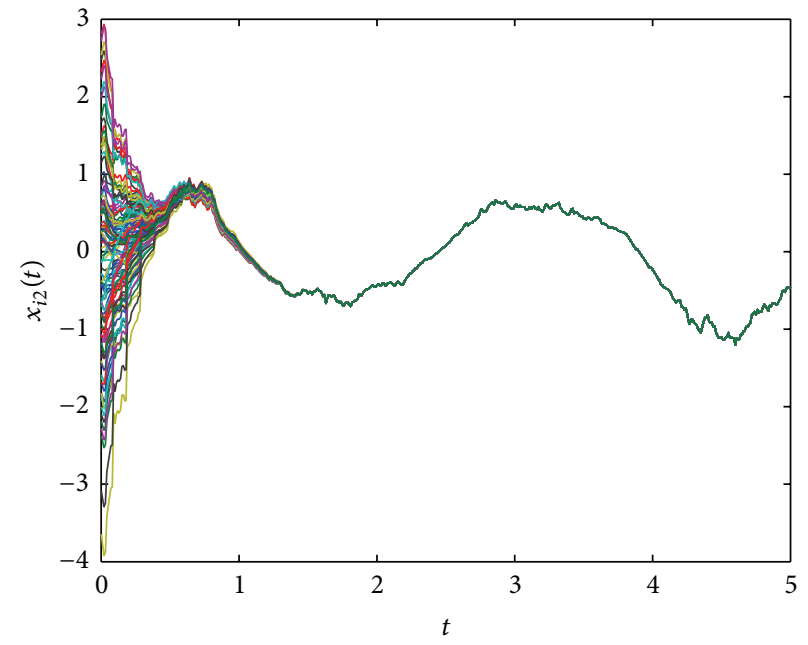

(b)

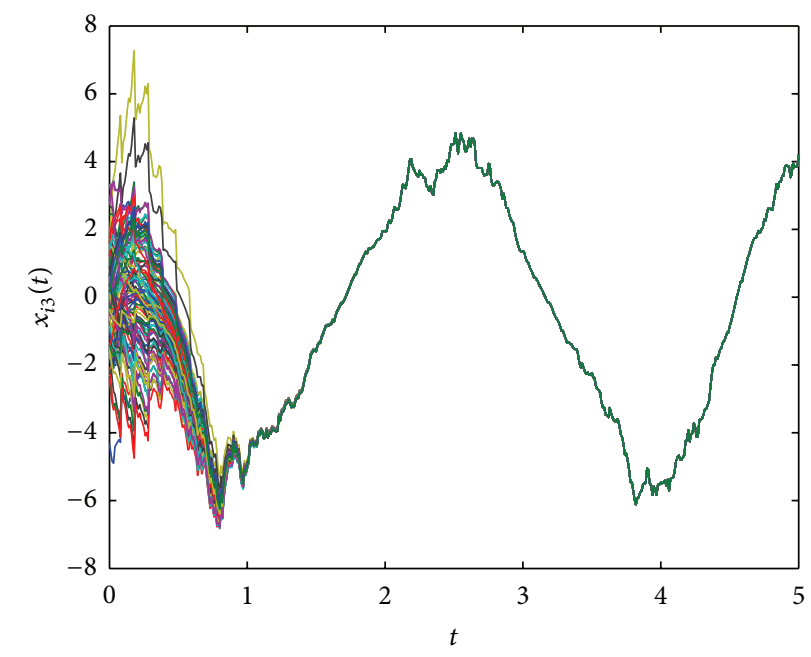

(c)

FIGURE 2: State variables $x_{i}(t), i=1,2, \ldots, 100$ of the coupled dynamical network with impulsive disturbances when $|\mu|<1$.

Similar procedures in (19) and (20) give

$$
\begin{aligned}
V\left(t_{k}^{+}\right) & =\sum_{i=1}^{N} e_{i}^{T}\left(t_{k}^{+}\right) e_{i}\left(t_{k}^{+}\right) \\
& =\mu^{2} \sum_{i=1}^{N} e_{i}^{T}\left(t_{k}^{-}\right) e_{i}\left(t_{k}^{-}\right)=\mu^{2} V\left(t_{k}^{-}\right),
\end{aligned}
$$

when $t=t_{k}, k \in N$.

By (36) and (39), we can obtain the following comparison system for the controlled impulsive stochastic dynamical Lur'e network (32):

$$
\begin{gathered}
\dot{w}(t)=\delta w(t) \quad t \geq 0, t \neq t_{k}, \\
w\left(t_{k}^{+}\right)=\mu^{2} w\left(t_{k}^{-}\right) \quad \mu^{2}>1, k \in N, \\
w\left(t_{0}\right)=E\left(V\left(t_{0}\right)\right) .
\end{gathered}
$$

Then, for any $t>0$, we have

$$
\begin{aligned}
w(t) & =e^{\delta\left(t-t_{0}\right)} \mu^{2 k} E\left(V\left(t_{0}\right)\right) \\
& =e^{\delta\left(t-t_{0}\right)} \mu^{2 N_{\zeta}\left(t, t_{0}\right)} E\left(V\left(t_{0}\right)\right) \\
& \leq e^{\delta\left(t-t_{0}\right)} \mu^{2\left(\left(t-t_{0}\right) / T_{a}\right)+2 N_{0}} E\left(V\left(t_{0}\right)\right) \\
& =\mu^{2 N_{0}} e^{\left(\left(2 \ln |\mu| / T_{a}\right)+\widetilde{\delta}\right)\left(t-t_{0}\right)} E\left(V\left(t_{0}\right)\right) .
\end{aligned}
$$

By Lemma 4, it can be concluded that the controlled impulsive stochastic dynamical Lur'e network (32) is exponentially stable, which can further imply that the dynamical network (7) can be exponentially stabilized to the objective trajectory $s(t)$ by a single controller on the first node. Theorem 13 is proved completely.

Remark 14. Considering when $|\mu|>1$, the term $2 \ln (|\mu|) / T_{a}$ is positive, it can be concluded from condition (34) in the Theorem 13 that the term $c \vartheta \gamma_{\min } \lambda_{\max }(\widetilde{Q})<0$ plays an 


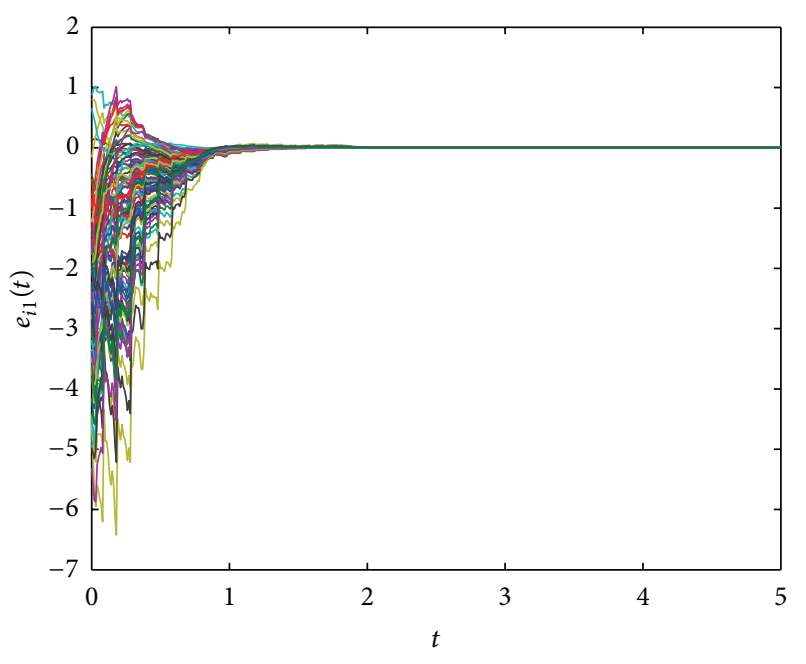

(a)

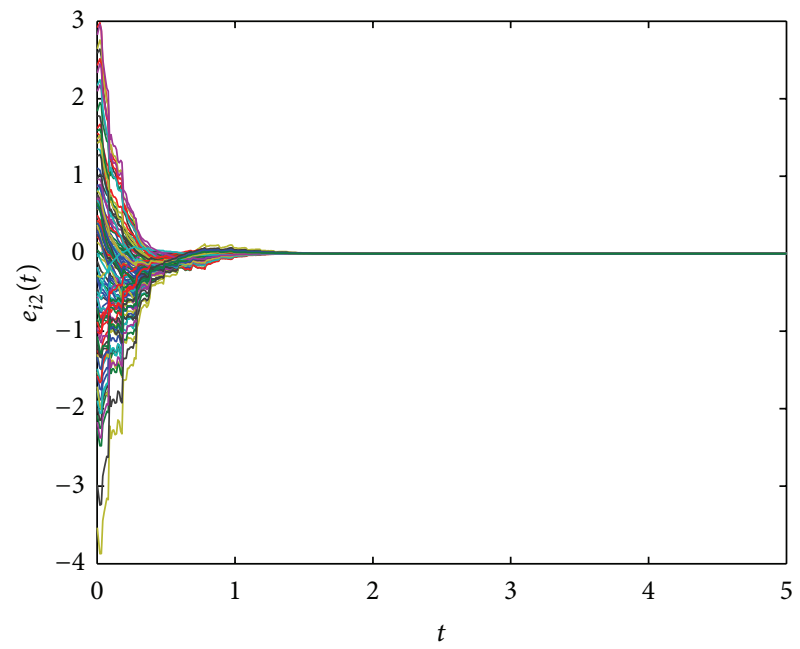

(b)

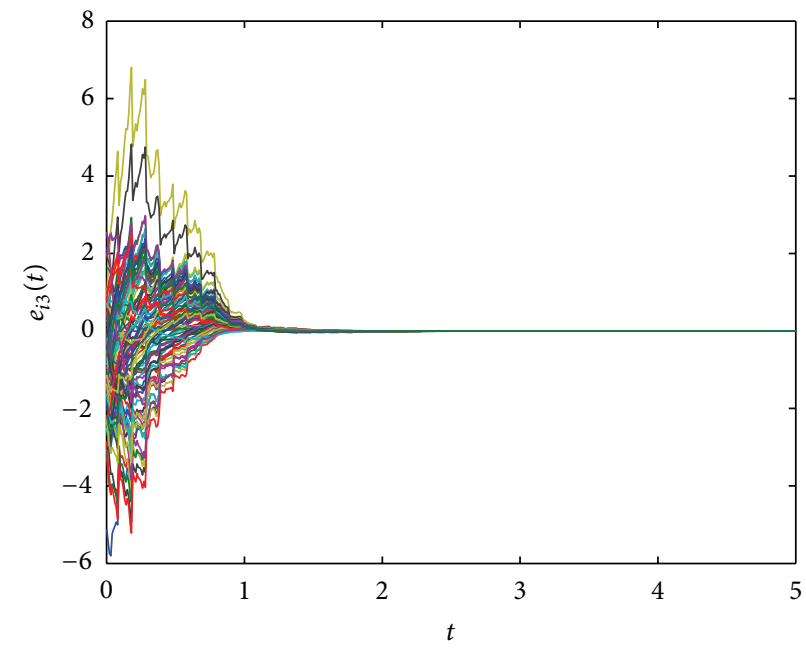

(c)

FIGURE 3: Error variables $e_{i}(t)=x_{i}(t)-s(t), i=1,2, \ldots, 100$ of the coupled dynamical network with impulsive disturbances when $|\mu|<1$.

important role for the synchronization of the network (7) under a single controller. The feedback controller is added to smooth the desynchronization effects caused by impulse.

\section{Numerical Simulation}

The above criteria can be applied to networks with different topologies and sizes. We present two examples with respect to two kinds of impulsive effects for illustrating the validity of the theory in the previous sections.

Example 15. We use Chua's circuit as one of a special case of the Lur'e system to verify the results in Theorem 8, namely, the impulsive effects are stabilizing. Chua's circuit has the following dimensionless equation:

$$
\left[\begin{array}{c}
\dot{x}_{1}(t) \\
\dot{x}_{2}(t) \\
\dot{x}_{3}(t)
\end{array}\right]=\left[\begin{array}{c}
\alpha\left(-x_{1}(t)+x_{2}(t)+g\left(x_{1}(t)\right)\right) \\
x_{1}(t)-x_{2}(t)+x_{3}(t) \\
-\beta x_{2}(t)-\gamma x_{3}(t)
\end{array}\right],
$$

where $g(y)=m_{1} y+(1 / 2)\left(m_{0}-m_{1}\right)(|y+1|-|y-1|) . \alpha, \beta$, $\gamma, m_{1}$, and $m_{0}$ are constants. Take $\alpha=10, \beta=15, \gamma=0.0385$, $m_{0}=1.27$, and $m_{1}=0.68$. Then, we have

$$
\begin{gathered}
x=\left[\begin{array}{l}
x_{1} \\
x_{2} \\
x_{3}
\end{array}\right], \quad A=\left[\begin{array}{ccc}
-3.2 & 10 & 0 \\
1 & -1 & 0 \\
0 & -15 & -0.0385
\end{array}\right], \\
B=\left[\begin{array}{c}
5.9 \\
0 \\
0
\end{array}\right], \quad C=\left[\begin{array}{lll}
1 & 0 & 0
\end{array}\right]
\end{gathered}
$$

and $\tilde{f}\left(C x_{1}(t)\right)=(1 / 2)\left(\left|x_{1}(t)+1\right|-\left|x_{1}(t)-1\right|\right)$. Considering the system (42) with (43), a chaotic attractor is plotted in Figure 1(a) with initial value $[0.1,0,0]$. 


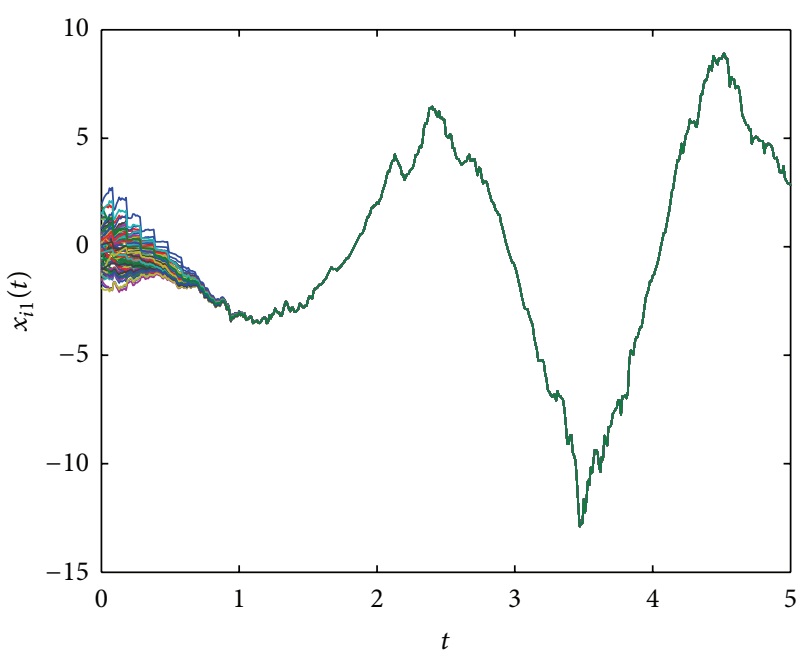

(a)

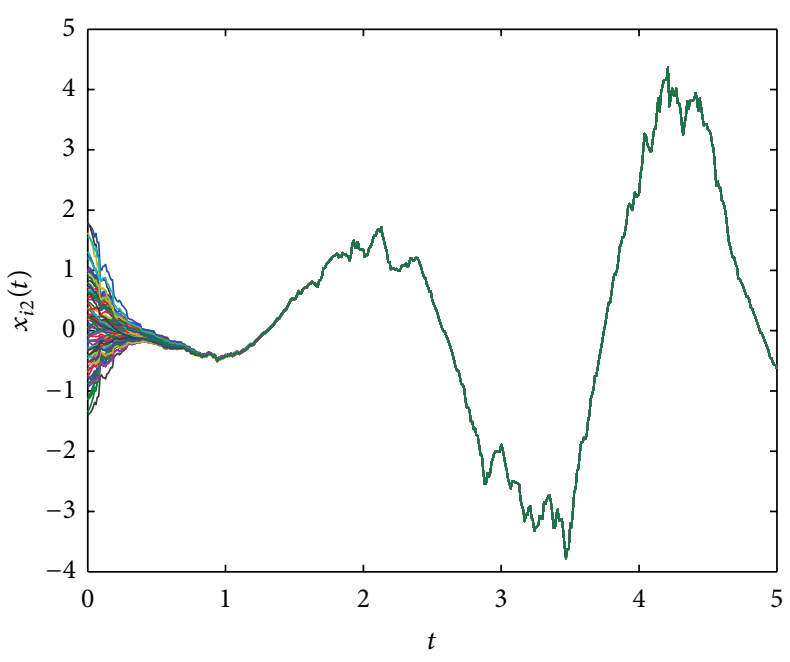

(b)

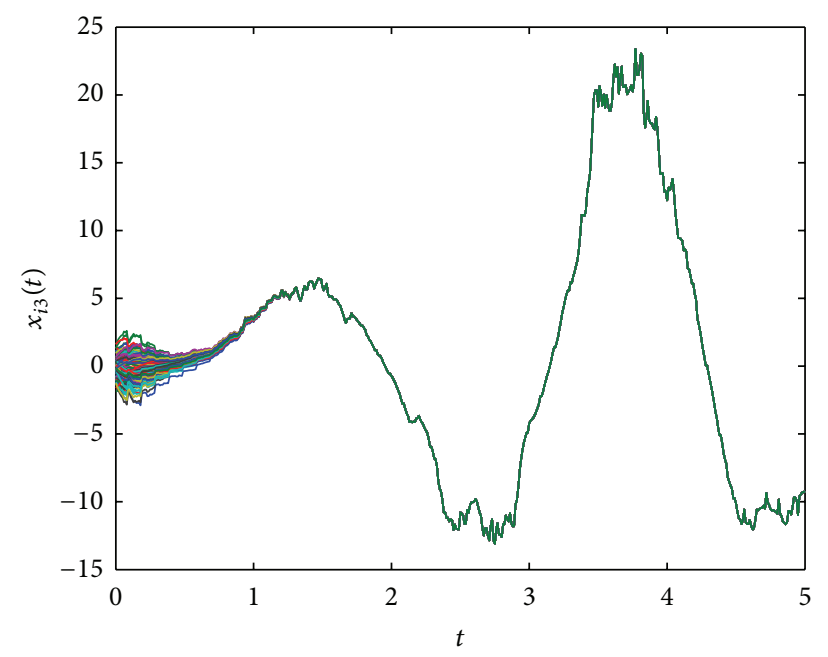

(c)

FIGURE 4: State variables $x_{i}(t), i=1,2, \ldots, 100$ of the coupled dynamical network with impulsive disturbances when $|\mu|>1$.

Chua's circuit with above permeates and Brownian noise are selected as the isolated node of the Lur'e dynamical network, and the $i$ th node is described as follows:

$$
\begin{aligned}
d x_{i}(t)= & {\left[A x_{i}(t)+B \tilde{f}\left(C x_{i}(t)\right)\right] d t } \\
& +\widetilde{g}\left(x_{i}(t), t\right) d w(t), \quad i=1,2, \ldots, 100,
\end{aligned}
$$

where $d w(t)$ is an $3 \mathrm{D}$ Brownian motion, the noise intensity function matrix $\widetilde{g}\left(x_{i}(t), t\right)=0.5 \cdot\left\|x_{i}(t)\right\| \cdot I_{3}$. Then, we have $\kappa=1$ for Assumption 6 and $M=0.5 \cdot I_{3}$ for Assumption 7 . We consider that a network consists of 100 nodes. The network structure is shown in Figure 1(b). Define the coupling matrix as follows: if there is a connection from nodes $i$ to $j$, then $l_{i j}=1$, otherwise $l_{i j}=0$. The nonlinear coupling function $h(\cdot)$ is selected as $h(x)=8 x+0.2 \sin (x)$ with $\vartheta=7$. Let the coupling strength $c=1$ and the inner coupling matrix $\Gamma=5 I_{3}$. Suppose that the average impulsive interval $T_{a}$ of the impulsive sequence is less than 0.1, because the impulsive strength $|\mu|<1$; here, we assume that $\mu=0.23$. By simple computation, it can be obtained that $2 \ln (|\mu|) / T_{a}=-29.3935$, $\delta=\lambda_{\max }\left(A+A^{T}+M^{T} M\right)+2 \kappa \sqrt{\lambda_{\max }\left(B^{T} B\right) \lambda_{\max }\left(C^{\mathrm{T}} C\right)}=$ 28.7250. Then, we can get that $2 \ln |\mu| / T_{a}+\lambda_{\text {max }}\left(A+A^{T}+\right.$ $\left.M^{T} M\right)+2 \kappa \sqrt{\lambda_{\max }\left(B^{T} B\right) \lambda_{\max }\left(C^{T} C\right)}<0$ for condition (13) in Theorem 8 .

We use $e_{i}(t)$ which stands for the error between $i$ th state and the synchronization goal $s(t)$, for $i=1,2,3$. Figure 2 is plotted to show the $i$ th variable state. The curves of the synchronization errors $e_{i}(t)$ at each state are plotted in Figure 3. It can be seen that the synchronization for the chaotic Chua's circuit is achieved because $e_{i}(t)$ converges to zero as $t \rightarrow+\infty$.

According to this example, one can get that the synchronization of the nonlinear and asymmetric impulsive stochastic Lur'e networks can be realized by applying a single impulsive controller. 


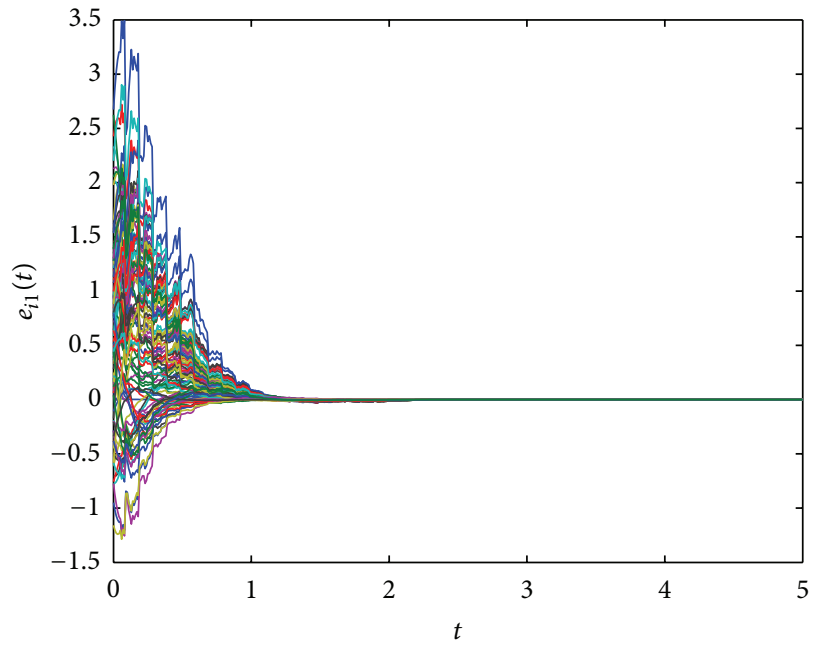

(a)

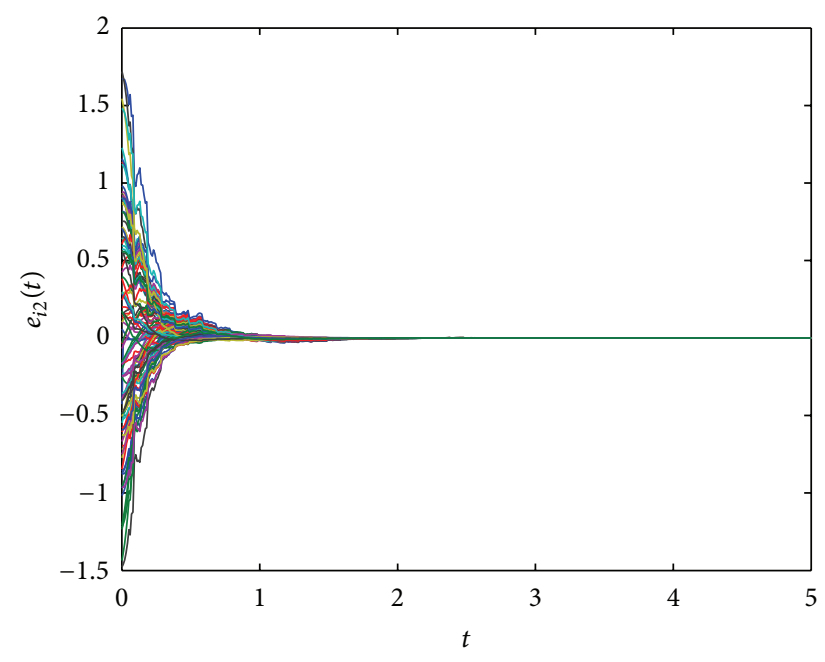

(b)

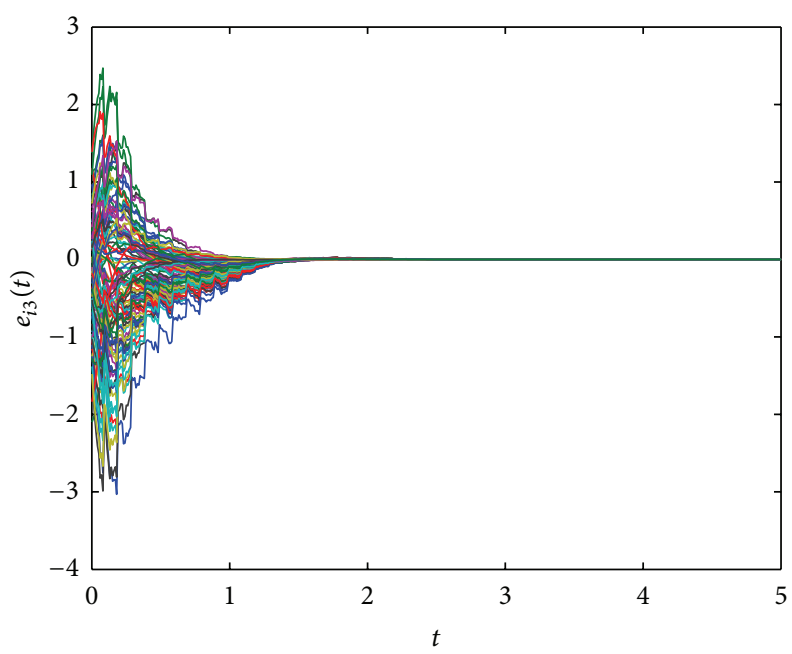

(c)

FIGURE 5: Error variables $e_{i}(t)=x_{i}(t)-s(t), i=1,2, \ldots, 100$ of the coupled dynamical network with impulsive disturbances when $|\mu|>1$.

Moreover, from Figure 3, it can be seen that the synchronization is achieved around $t=1$. Compared with the numerical results obtained in [12], in which the synchronization is reached near $t=0.2$, we could conjecture that the stochastic term in (8), which is absent in the studies of [12], could slow down the speed of synchronization.

Example 16. We still use the complex network built in Example 15 with $|\mu|=1.1$ to verify the results in Theorem 13, namely, the impulsive effects are destabilizing. We choose the coupling strength $c=87.68$. Calculation shows that $\delta=\lambda_{\text {max }}\left(A+A^{T}+M^{T} M\right)+2 \kappa \sqrt{\lambda_{\text {max }}\left(B^{T} B\right) \lambda_{\text {max }}\left(C^{T} C\right)}+$ $c \vartheta \gamma_{\min } \lambda_{\max }(\widetilde{Q})=-0.2587$ and $2 \ln (|\mu|) / T_{a}=0.1906$.

We use $e_{i}(t)$ which stands for the error between $i$ th state and the synchronization goal $s(t)$, for $i=1,2,3$. Figure 4 is plotted to show the $i$ th variable state. The curves of the synchronization errors $e_{i}(t)$ at each state are plotted in Figure 5. It can be seen that the synchronization for the chaotic Chua's circuit is achieved because $e_{i}(t)$ converges to zero as $t \rightarrow+\infty$.

Compared with Figures 3 and 5, it can be seen that both of them achieve synchronization around $t=1$. However, it should be pointed out that since the impulsive effects are destabilizing, the coupling strength is chosen to be $c=87.68$, which is much larger than $c=1$ in Example 15. Moreover, a single feedback controller is added in (32), which reflects the influence of the destabilizing impulsive effects.

\section{Conclusion}

In this paper, we investigated the impulsive stochastic dynamical network models with nonlinear and asymmetrical couplings. The nodes which consisted of the network are the Lur'e systems, and the dynamics of each node is nonlinear. The manners of communication between nodes are impulsive at 
some certain impulsive instants, and interconnect normally at nonimpulsive instants. Due to the instantaneous perturbations and abrupt change at certain instants, the stochastic terms are considered when we analyze the stability. We transmitted the synchronization problem into proving the stability of the error system. By using the pinning control scheme, we applied a single impulsive controller to the Lur'e network for stabilizing impulsive effects and destabilizing impulsive effects, respectively. It was proved as an effective way for the realization of the global synchronization. We also made use of two examples to illustrate the theoretical analysis.

\section{Acknowledgments}

The authors thank the referees and the editor for their valuable comments on this paper. This work is supported by the Natural Science Foundation of China (Grant no. 61273220), Guangdong Education University Industry Cooperation Projects (Grant no. 2009B090300355), the Shenzhen Basic Research Project (JC201006010743A, JCYJ20120613105730482), and the 2011 Foundation for Distinguished Young Talents in Higher Education of Guangdong (LYM11115).

\section{References}

[1] S. H. Strogatz and I. Stewart, "Coupled oscillators and biological synchronization," Scientific American, vol. 269, no. 6, pp. 102109, 1993.

[2] C. M. Gray, "Synchronous oscillations in neuronal systems: mechanisms and functions," Journal of Computational Neuroscience, vol. 1, no. 1-2, pp. 11-38, 1994.

[3] J. Fell and N. Axmacher, "The role of phase synchronization in memory processes," Nature Reviews Neuroscience, vol. 12, no. 2, pp. 105-118, 2011.

[4] W. He, F. Qian, J. Cao, and Q.-L. Han, "Impulsive synchronization of two nonidentical chaotic systems with time-varying delay," Physics Letters A, vol. 375, no. 3, pp. 498-504, 2011.

[5] L. M. Pecora and T. L. Carroll, "Synchronization in chaotic systems," Physical Review Letters, vol. 64, no. 8, pp. 821-824, 1990.

[6] H. Li, B. Chen, Q. Zhou, and W. Qian, "Robust stability for uncertain delayed fuzzy Hopfield neural networks with Markovian jumping parameters," IEEE Transactions on Systems, Man, and Cybernetics B, vol. 39, no. 1, pp. 94-102, 2009.

[7] H. Li, H. Gao, and P. Shi, "New passivity analysis for neural networks with discrete and distributed delays," IEEE Transactions on Neural Networks, vol. 22, no. 11, pp. 1842-1847, 2010.

[8] A. Pikovsky, M. Rosenblum, and J. Kurths, Synchronization: A Universal Concept in Nonlinear Sciences, vol. 12, Cambridge University Press, Cambridge, UK, 2001.

[9] Y. Tang, H. Gao, W. Zou, and J. Kurths, "Identifying controlling nodes in neuronal networks in different scales," PLOS ONE, vol. 7, Article ID e41375, 2012.

[10] Y.-W. Wang, H. O. Wang, J.-W. Xiao, and Z.-H. Guan, "Synchronization of complex dynamical networks under recoverable attacks," Automatica, vol. 46, no. 1, pp. 197-203, 2010.

[11] J. Q. Lu, Z. D. Wang, J. D. Cao, D. W. C. Ho, and J. Kurths, "Pinning impulsive stabilization of nonlinear dynamical networks with time-varying delay," International Jorurnal of Bifurcation and Chaos, vol. 22, no. 7, Article ID 1250176, 12 pages, 2012.
[12] J. Lu, D. W. C. Ho, J. Cao, and J. Kurths, "Single impulsive controller for globally exponential synchronization of dynamical networks," Nonlinear Analysis: Real World Applications, vol. 14, no. 1, pp. 581-593, 2013.

[13] J. Q. Lu, J. Kurths, J. D. Cao, N. Mahdavi, and C. Huang, "Synchronization control for nonlinear stochastic dynamical networks: pinning impulsive strategy," IEEE Transactions on Neural Networks and Learning Systems, vol. 23, no. 2, pp. 285-292, 2012.

[14] J. Lu, D. W. C. Ho, J. Cao, and J. Kurths, "Exponential synchronization of linearly coupled neural networks with impulsive disturbances," IEEE Transactions on Neural Networks, vol. 22, no. 2, pp. 329-335, 2011.

[15] G. Zhang, Z. Liu, and Z. Ma, "Synchronization of complex dynamical networks via impulsive control," Chaos, vol. 17, no. 4, Article ID 043126, 9 pages, 2007.

[16] B. Liu, X. Liu, G. Chen, and H. Wang, "Robust impulsive synchronization of uncertain dynamical networks," IEEE Transactions on Circuits and Systems I, vol. 52, no. 7, pp. 1431-1441, 2005.

[17] B. C. Goodwin, "Oscillatory behavior in enzymatic control processes," Advances in Enzyme Regulation, vol. 3, pp. 425-428, 1965.

[18] M. B. Elowitz and S. Leibier, "A synthetic oscillatory network of transcriptional regulators," Nature, vol. 403, no. 6767, pp. 335$338,2000$.

[19] T. S. Gardner, C. R. Cantor, and J. J. Collins, "Construction of a genetic toggle switch in Escherichia coli," Nature, vol. 403, no. 6767, pp. 339-342, 2000.

[20] V. Gazi and K. M. Passino, "Stability analysis of social foraging swarms," IEEE Transactions on Systems, Man, and Cybernetics $B$, vol. 34, no. 1, pp. 539-557, 2004.

[21] L. O. Chua, "Chuas circuit: an overview ten years later," Journal of Circuits, Systems and Computers, vol. 4, no. 117, pp. 117-159, 1994.

[22] W. He, F. Qian, Q. L. Han, and J. Cao, "Synchronization error estimation and controller design for delayed Lure systems with parameter mismatches," IEEE Transactions on Neural Networks and Learning Systems, vol. 23, no. 10, pp. 1551-1563, 2012.

[23] X. Huang, J. Cao, and D. W. C. Ho, "Synchronization criteria for Lur'e systems by dynamic output feedback with time-delay," International Journal of Bifurcation and Chaos in Applied Sciences and Engineering, vol. 16, no. 8, pp. 2293-2307, 2006.

[24] J. Cao, H. X. Li, and D. W. C. Ho, "Synchronization criteria of Lur'e systems with time-delay feedback control," Chaos, Solitons and Fractals, vol. 23, no. 4, pp. 1285-1298, 2005.

[25] M. E. Yalçin, J. A. K. Suykens, and J. Vandewalle, "Master-slave synchronization of Lur'e systems with time-delay," International Journal of Bifurcation and Chaos in Applied Sciences and Engineering, vol. 11, no. 6, pp. 1707-1722, 2001.

[26] X. Liu, J. Wang, and L. Huang, "Global synchronization for a class of dynamical complex networks," Physica A, vol. 386, no. 1, pp. 543-556, 2007.

[27] T. Wang, T. Li, X. Yang, and S. M. Fei, "Cluster synchronization for delayed Lur'e dynamical networks based on pinning control," Neurocomputing, vol. 83, no. 15, pp. 72-82, 2012.

[28] J. Cao, Z. Wang, and Y. Sun, "Synchronization in an array of linearly stochastically coupled networks with time delays," Physica A, vol. 385, no. 2, pp. 718-728, 2007.

[29] J. Lu, D. W. C. Ho, and J. Cao, "A unified synchronization criterion for impulsive dynamical networks," Automatica, vol. 46, no. 7, pp. 1215-1221, 2010. 
[30] C. W. Wu and L. O. Chua, "Synchronization in an array of linearly coupled dynamical systems," IEEE Transactions on Circuits and Systems I, vol. 42, no. 8, pp. 430-447, 1995.

[31] B. Liu, "Stability of solutions for stochastic impulsive systems via comparison approach," IEEE Transactions on Automatic Control, vol. 53, no. 9, pp. 2128-2133, 2008. 


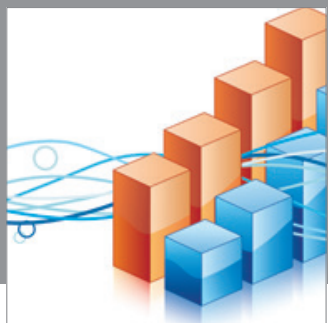

Advances in

Operations Research

mansans

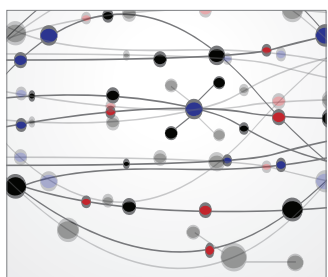

The Scientific World Journal
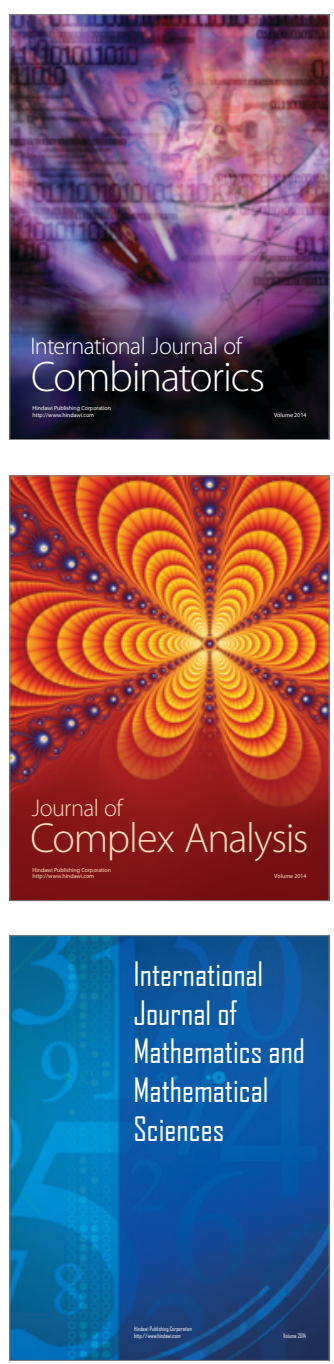
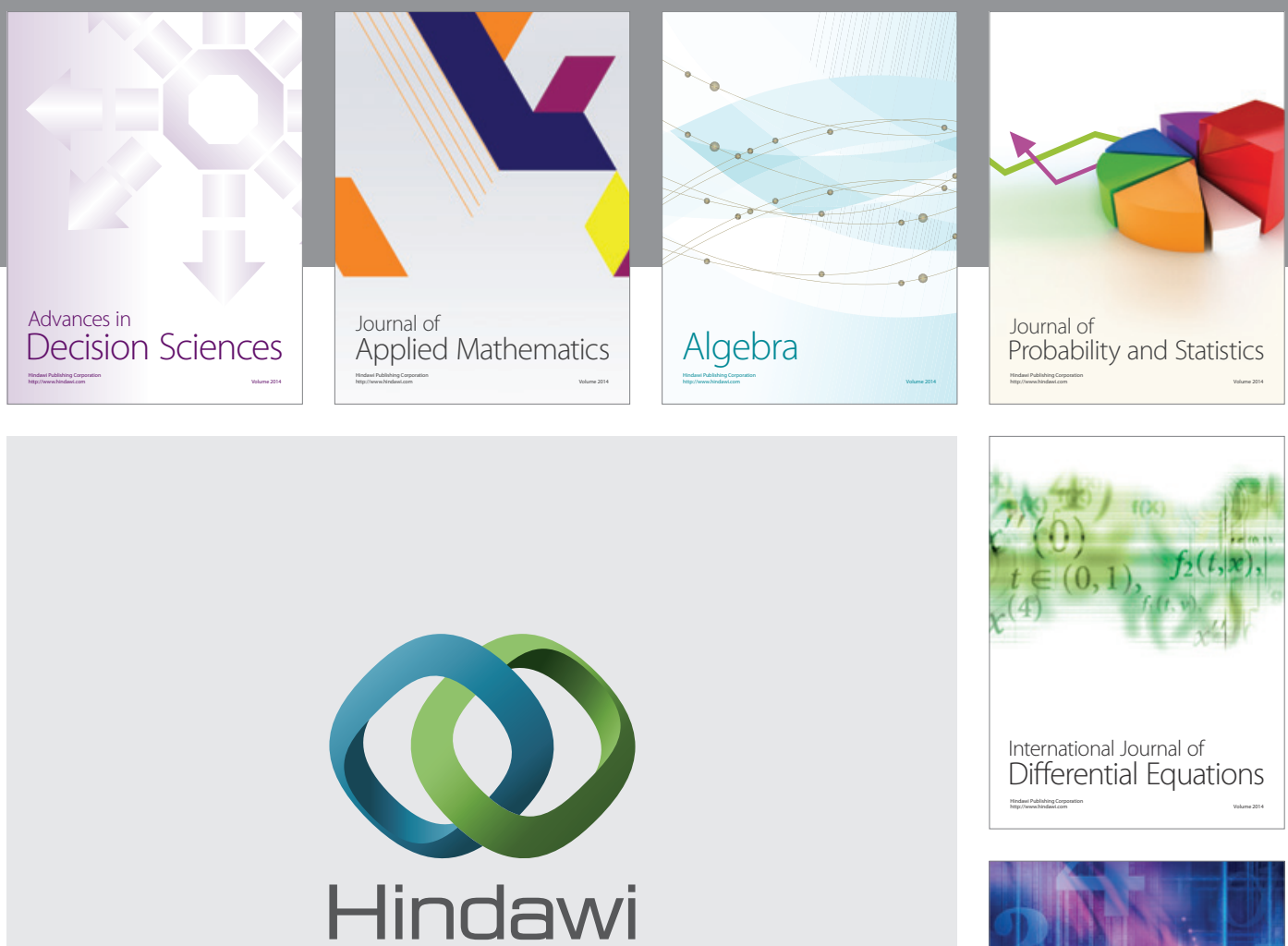

Submit your manuscripts at http://www.hindawi.com
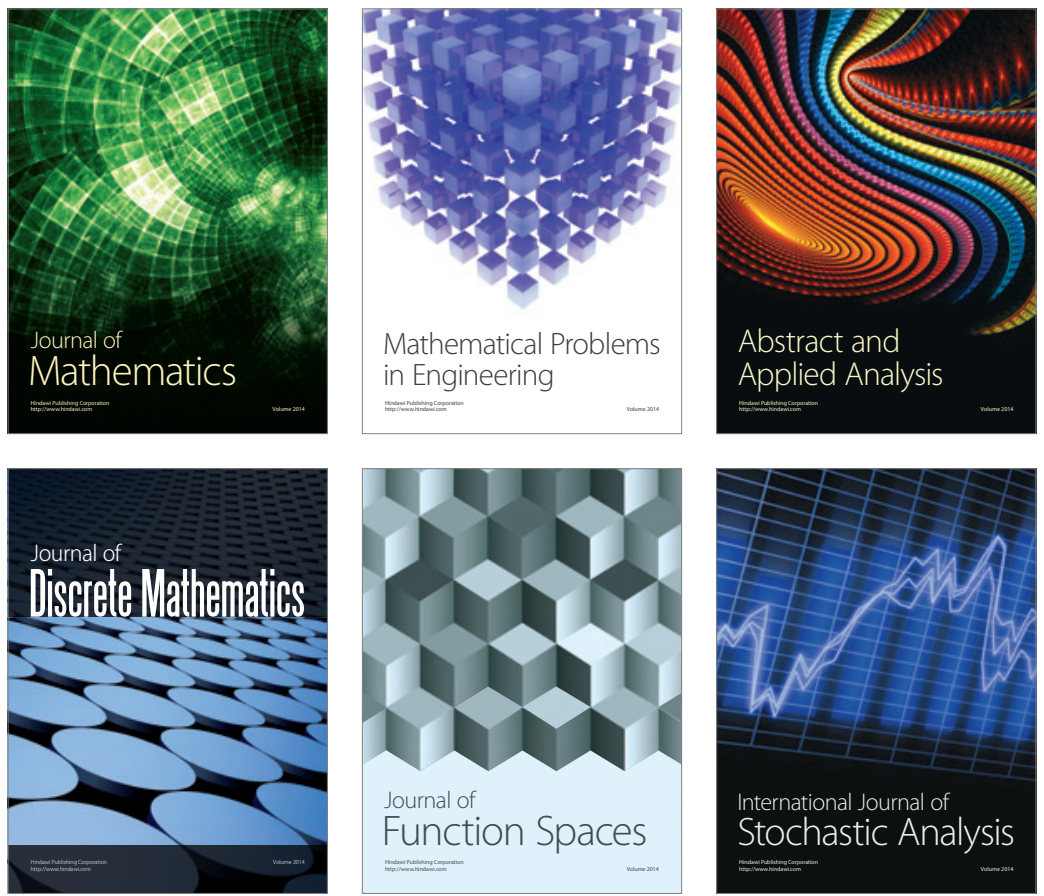

Journal of

Function Spaces

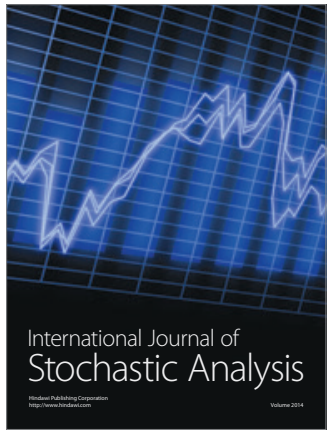

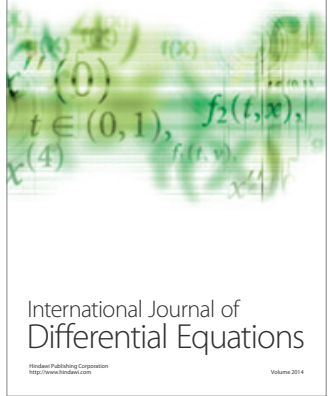
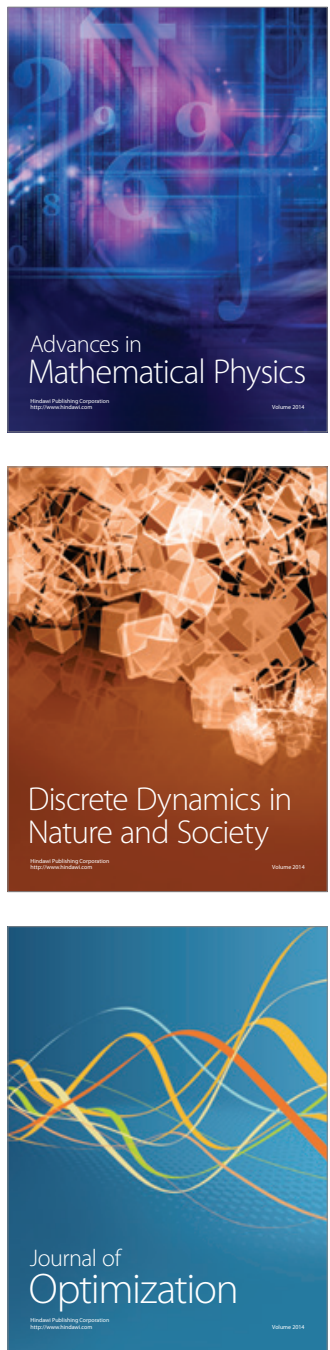